\title{
Virtual assessment of damage detection techniques for operational wind turbine.
}

\author{
Emilio Di Lorenzo, Simone Manzato, Bart Peeters, Herman Van der \\ Auweraer.
}

LMS International, RTD Test Division, Interleuvenlaan 68, 3001, Leuven, Belgium

\begin{abstract}
Operational Modal Analysis (OMA), also known as output-only modal analysis, allows identifying modal parameters only by using the response measurements of the structures in operational conditions when the input forces cannot be measured. These information can then be used to improve numerical models in order to monitor the operating and structural conditions of the system. This is a critical aspect both for condition monitoring and maintenance of large wind turbines, particularly in the off-shore sector where operation and maintenance represent a high percentage of total costs. Although OMA is widely applied, the wind turbine case still remains an open issue. Numerical aeroelastic models could be used, once they have been validated, to introduce virtual damages to the structures in order to analyze the generated data. Results from such models can then be used as baseline to monitor the operating and structural condition of the machine.
\end{abstract}

\section{Introduction}

The objective of this paper is to apply advanced Operational Modal Analysis (OMA) techniques to predict in advance failures or damages with a monitoring application based on the analysis of modal parameters and their variations in operating conditions. In this way, the risk to have catastrophic failures and cost associated can be significantly reduced. OMA is a technique that allows extracting the modal parameters from vibration response signals. The main difference compared to the traditional experimental modal analysis is that it does not need the measurement of the input forces so that also structures under operating conditions, or in other situations where it is impossible to measure the input forces, can be tested. The information obtained from this analysis can then be used to improve numerical models, to predict the dynamic behavior of new designs, to identify the modal parameters of prototypes and to monitor systems in operating conditions.

The problem associated with the dynamic identification of wind turbines has its roots in 1990 when a special technique known as Natural Excitation Technique (NExT) was developed to estimate modal parameters of wind turbines excited in their operating environment [1]. In the following years, this technique has been 
applied to other fields such as civil structures as well as automotive and aerospace applications. Although the first application of an Operational Modal Analysis methodology was related to a vertical-axis wind turbine, not many other applications to wind turbines were studied later on. The main reason is the fact that most of the OMA assumptions are violated by operating wind turbines.

With this paper, an advanced OMA technique, the so-called PolyMAX Operational Modal Analysis technique is applied to different set of simulated data obtained introducing virtual damages to the unit under test. The main objective is to predict damages by means of modal parameters variations for condition monitoring and maintenance (O\&M) purposes. In section 2 the PolyMAX Operational Modal Analysis technique is briefly presented. The NREL offshore 5-MW baseline wind turbine is sketched out in Section 3. Section 4 describes the virtual damages introduced in the wind turbine such as ice on all the blades and unbalanced masses. Finally in Section 5 the mentioned OMA technique is applied to the different load cases.

\section{PolyMAX Operational Modal Analysis}

Operational modal analysis has attracted a significant amount of research interest in the past years. Several operational modal analysis techniques such as Frequency Domain Decomposition (FDD), Stochastic Subspace Identification (SSI) and Operational PolyMAX have been developed and evaluated [2]. In this paper, the PolyMAX method is selected to perform the operational modal analysis. It has been developed as a polyreference version of the least-squares complex frequency-domain (LSCF) estimation method using a so-called right matrix-fraction model. This method, in case of OMA, requires output spectra as primary data. It can be demonstrated that, under the assumption of white noise input, output spectra can be modeled very similarly to FRFs [3].

In case of Experimental Modal Analysis [4], the modal decomposition of an FRF matrix $[\mathrm{H}(\omega)]$ is:

$$
[H(\omega)]=\sum_{i=1}^{N} \frac{\left\{v_{i}\right\}\left\langle l_{i}^{T}\right\rangle}{j \omega-\lambda_{i}}+\frac{\left\{v_{i}^{*}\right\}\left\langle l_{i}^{H}\right\rangle}{j \omega-\lambda_{i}^{*}}
$$

where 1 is the number of outputs; $\mathrm{N}$ is the number of modes and half of the system order, ${ }^{*}$ is the complex conjugate operator, ${ }^{\mathrm{H}}$ is the complex conjugate transpose of a matrix, $\left\{v_{i}\right\}$ are the mode shapes, $\left\langle l_{i}^{T}\right\rangle$ are the modal participation factors and $\lambda_{i}$ are the poles. The system poles are recurring in complex-conjugate pairs and are related to the eigenfrequencies $\omega_{i}$ and damping ratios $\xi_{i}$ as: 


$$
\lambda_{i}, \bar{\lambda}_{i}=-\xi_{i} \omega_{i} \pm j \sqrt{1-\xi_{i}^{2}} \cdot \omega_{i}
$$

Now, the input spectra $\left[\mathrm{S}_{\mathrm{uu}}(\omega)\right]$ and output spectra $\left[\mathrm{S}_{\mathrm{yy}}(\omega)\right]$ of a system represented by the FRF matrix in Equation (1) are related as:

$$
\left[S_{y y}(\omega)\right]=[H(\omega)]\left[S_{\text {ии }}(\omega)\right][H(\omega)]^{H}
$$

In case of operational data, output spectra are the only available information. The deterministic knowledge of the input is replaced by the assumption that the input is white noise, which is characterized by a constant power spectrum and is independent of the frequency. The modal decomposition of the output spectrum matrix can be obtained now by inserting Equation (1) in Equation (3) and converting to partial fraction form:

$$
\left[S_{y y}(\omega)\right]=\sum_{i=1}^{N} \frac{\left\{v_{i}\right\}\left\langle g_{i}\right\rangle}{j \omega-\lambda_{i}}+\frac{\left\{v_{i}^{*}\right\}\left\langle g_{i}^{*}\right\rangle}{j \omega-\lambda_{i}^{*}}+\frac{\left\{g_{i}\right\}\left\langle v_{i}\right\rangle}{-j \omega-\lambda_{i}}+\frac{\left\{v_{i}\right\}\left\langle g_{i}\right\rangle}{-j \omega-\lambda_{i}^{*}}
$$

where $\left\langle g_{i}\right\rangle$ are the so-called operational reference factors, which replace the modal participation factors in case of output-only data. Their physical interpretation is less obvious as they are a function of all modal parameters of the system and the constant input spectrum matrix. The main goal of OMA will then be to identify the right-end side of Equation (4) by using measured output data preprocessed into output spectra.

The PolyMAX algorithm greatly facilitates the operational modal parameter estimation process by producing extremely clear stabilization diagrams, making the pole selection a lot easier by means of estimating unstable poles (i.e. mathematical or noise modes) with negative damping making them relatively easy to separate from the stable poles (i.e. system modes).

The PolyMAX technique has been widely employed for OMA of civil engineering and mechanical structures including bridges, a football stadium [5], a satellite and many others [6]. It has also been computationally optimized to analyze large data sets with a broad frequency band up to high model orders. In the next sections, the Operational PolyMAX method is applied to a 5MW wind turbine in several conditions.

\section{NREL offshore 5MW baseline wind turbine model}

In this section the virtual wind turbine model used as test case is described. The NREL offshore 5-MW baseline wind turbine has been developed by the National Renewable Energy Laboratory (NREL) to support concept studies aimed at as- 
sessing offshore wind technology. It is a conventional three-bladed upwind variable-speed variable blade-pitch-to-feather-controlled turbine [7].
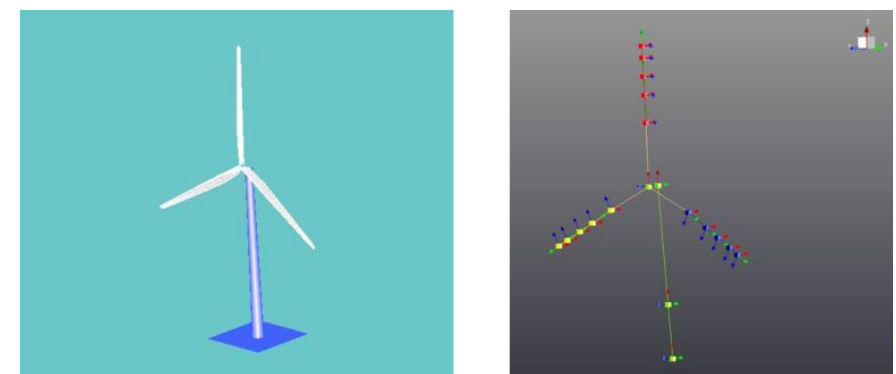

Figure 1: NREL 5 MW S4WT model (left) and Test.Lab geometry (right).

The main objective is to analyze the global dynamic behavior of the full-scale turbine; for this reason, the model has been built as simple as possible. The generated model is shown in Figure 1 and it can be divided in three main components:

- Tower: it is modeled as 5 elastic beam elements with lumped masses and hinged to the ground foundation. The total tower height is $90 \mathrm{~m}$.

- Rotor: in the 3-bladed rotor, each blade is identical and is modeled with $17 \mathrm{sec}$ tions with specific mass, elastic and aerodynamic properties.

- Drivetrain: the transmission is simplified into a 1 degree-of-freedom system with a gear-ratio of 97 between the Low Speed Shaft (LSS) and the High Speed Shaft (HSS). The generator torque is regulated by the controller model.

The software SAMCEF for Wind Turbines (S4WT) allows the user to define both a structural and an aerodynamic model which are then solved together to obtain the coupled aero-elastic solution [8]. Different parameters can be assigned; in this analysis a turbulent wind has been applied because the interest of the analysis lays in the turbine dynamic response in real conditions. The wind dominant component is in the X direction (in the model, from LSS to HSS), but to have a more realistic response also turbulent fluctuations on the other two directions are included.

Table 1: Wind turbine main parameter.

\begin{tabular}{ll}
\hline Blade Length & $61.5 \mathrm{~m}$ \\
\hline Blade Overall Mass & $17740 \mathrm{Kg}$ \\
Tower Height above Ground & $87.6 \mathrm{~m}$ \\
Tower Overall Mass & $347460 \mathrm{~kg}$ \\
Hub Mass & $56780 \mathrm{~kg}$ \\
Nacelle Mass & $240000 \mathrm{~kg}$ \\
Gearbox Ratio & $97: 1$ \\
\hline
\end{tabular}




\section{Load cases}

The model of the wind turbine is analyzed in different operating conditions and the modal parameters are extracted from the generated acceleration signals by means of Operational Modal Analysis. The different models are solved in S4WT and accelerations are computed and exported in LMS Test.Lab for processing and identification[9].

In order to have simulated accelerations that can be considered as accelerations obtained from tri-axial accelerometers mounted on the blades, it is necessary to consider them in the local reference frame in which the $\mathrm{X}$ axis is the blade axis (oriented toward the blade tip), the $\mathrm{Y}$ axis is aligned with the chord-line and belongs to the blade section plane (oriented toward the leading edge) and the $\mathrm{Z}$ axis is normal to the chord line and belongs to the blade section planes. Using this axis configuration, the edge-wise modes are described by bending along the $\mathrm{Y}$ axis while flap-wise modes bend the structure along the $\mathrm{Z}$ axis. Axial modes along the blade pitch axis can be neglected since they appear at frequencies much higher than those the analysis will focus on in this paper.

The locations selected to measure the accelerations are:

- 3 sensors distributed along the tower

- 1 sensor at the hub center

- 5 sensors per-blade located on the pitch axis

After analyzing the response of the structure in reference and ideal conditions, different possible damages will be introduced to understand how they affect the measured accelerations. In this preliminary assessment, two main damages are introduced:

- Blade icing

- Mass unbalance on a blade

The presence of ice on the blades can create excessive turbine vibration and can change the natural frequencies of the blades as well as increase the fatigue loads. It is very important to predict when the icing phenomena occur. Icing has two main effects [10]; on one hand it modifies the blade shape increasing the drag and decreasing the lift and on the other hand the presence of an additional and not uniformly distributed mass could cause unbalancing of the rotor. Effect of ice is not only related to performance issues, but also to safety ones; in fact, during the operational conditions, lumps of ice can detach from the blade and cause damage to people or things.

Within the software S4WT, the conditions "ice formation on all rotor blades" and "ice formation on all rotor blades except one" can be investigated. Calculation is based on the guidelines for certification of wind turbines [11], where it is suggested that the mass distribution (mass/unit length) is assumed at the leading edge of the rotor blade and it increases linearly from zero at the rotor axis to the maxi- 
mum value $\mu_{\mathrm{E}}$ at half the radius and then it remains constant up to the outermost radius. The value $\mu_{\mathrm{E}}$ is calculated as:

$$
\begin{aligned}
& \mu_{E}=\rho_{E} \cdot k \cdot c_{\text {min }} \cdot\left(c_{\text {max }}+c_{\text {min }}\right) \\
& k=0.00675+0.3 \exp (-0.32 \cdot R)
\end{aligned}
$$

where ${ }^{\mu_{E}}$ is the mass distribution on the leading edge of the rotor blade at half of the rotor radius, $\rho_{E}$ is the ice density, $\mathrm{R}$ is the rotor radius, $\mathrm{c}_{\max }$ is the maximum chord length and $\mathrm{c}_{\min }$ is the length at the blade tip, linearly extrapolated from the blade contour.

The second damage condition which was simulated is the presence of an unbalanced mass that is added to a particular location on the wind turbine. A little cubic mass, which properties can be found in the Table 2, was considered for this preliminary analysis.

Table 2: Unbalanced mass properties.

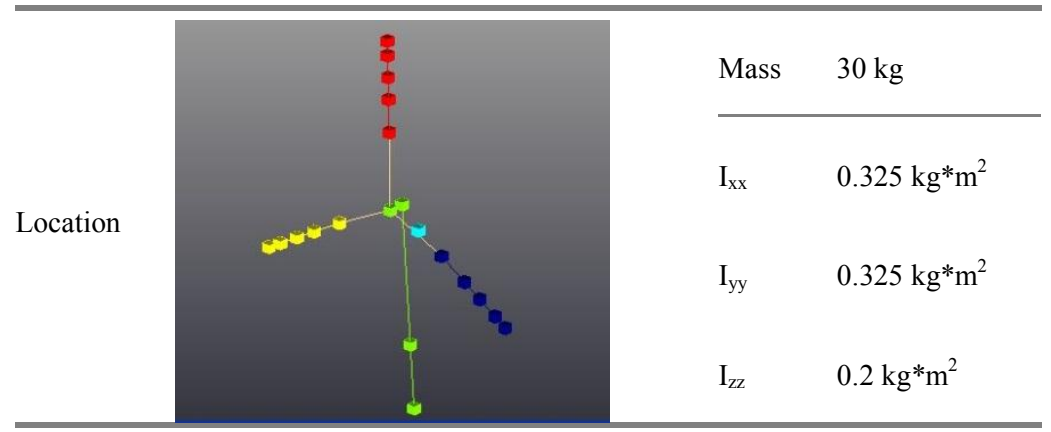

Below, the different operating conditions investigated in this paper are summarized:

- Reference parked conditions.

- Parked conditions with ice on all blades.

- Parked conditions on all blades but one.

- Reference power production.

- Power production with ice on all blades.

- Power production with ice on all blades but one.

- Power production with unbalanced mass. 


\section{Data analysis}

First of all, the wind turbine is analyzed in parked conditions. This load case represents the condition in which the blades are parked and the generator is disconnected; the first seconds are used to place the pitch in its parking position and the rotor at the angle specified as initial rotor angle. At the start time, the rotor is released, but the pitch remains fixed.

To perform Operational Modal Analysis, the point at the tower top is chosen as reference for the correlations and spectra computation, which are then used to extract the modal parameters by means of PolyMAX method [12]. To simplify the entire procedure, the accelerations along the blade axis are neglected. The identified modes are then compared to those one obtained using both the FAST model and the ADAMS model; in FAST the natural frequencies are calculated by performing an eigenanalysis on the first-order matrix created from a linearization analysis, while in ADAMS a command that linearizes the complete model and compute eigenparameters is used.

Table 3: Numerical modes in parked conditions: STS: side-to-side, FA for-aft; blade modes are described based on their main motion orientation.

\begin{tabular}{lllll}
\hline \multirow{2}{*}{ Mode } & Description & Natural frequencies $[\mathrm{Hz}]$ & \\
\cline { 3 - 5 } & & Test.Lab & FAST & ADAMS \\
1 & $1^{\text {st }}$ Tower STS & 0.312 & 0.312 & 0.319 \\
2 & $1^{\text {st }}$ Tower FA & 0.329 & 0.324 & 0.316 \\
3 & $1^{\text {st }}$ Flap Yaw & 0.666 & 0.666 & 0.630 \\
4 & $1^{\text {st }}$ Flap Pitch & 0.675 & 0.668 & 0.669 \\
5 & $1^{\text {st }}$ Flap Sym & 0.720 & 0.700 & 0.702 \\
6 & $1^{\text {st }}$ Edge Pitch & 1.056 & 1.079 & 1.074 \\
7 & $1^{\text {st }}$ Edge Yaw & 1.059 & 1.089 & 1.088 \\
8 & $2^{\text {nd }}$ Flap Yaw & 1.853 & 1.934 & 1.651 \\
9 & $2^{\text {nd }}$ Flap Pitch & 1.888 & 1.922 & 1.856 \\
10 & $2^{\text {nd }}$ Flap Sym & 1.900 & 2.021 & 1.960 \\
\hline
\end{tabular}

The results are summarized in Table 3 and the agreement between them is quite good. By using PolyMAX all the first 10 modes can be identified and the biggest differences exist in the predictions of the blades second asymmetric flapwise yaw and pitch modes. "Yaw" and "pitch" mean that these blade asymmetric modes couple with the nacelle-yaw and nacelle-pitching motions, respectively.

The natural frequencies shown in Table 3 are obtained in the standard configuration; they can be compared to those one obtained in other cases such as that one in which the presence of ice is simulated on all the blades or that one in which the ice is on all the blades but one. In order to perform a better analysis, the point at the blade root is chosen as reference for the correlations and spectra computation and the blade axis accelerations are neglected. The results, in the form of frequen- 
cies and damping values, are shown in Table 4 for some of the modes. A frequency shift due to the added ice mass on the blades can be observed and, as expected, increasing the mass decreases the natural frequencies. Not so many considerations can be done regarding the damping values because a global trend cannot be seen moving from one condition to another one, but it should be analyzed mode by mode. Besides, possible effect on damping should be more visible in operating conditions. During ice events, ice accumulates on the rotor blades modifying the blade shape and reducing the aerodynamic efficiency. The amount by which the lift decreases and the drag increases depends on the quality, shape and position of the ice. By using S4WT, the blade shape modification cannot be simulated from an aerodynamic side and a comparison between experimental and simulated data is still possible from a frequency shift point of view, but not from a damping variation point of view where experimental and simulation results can be quite different [13].

Table 4: Numerical modes in parked conditions for the different analyzed configurations.

\begin{tabular}{llll}
\hline Mode & Standard configuration & Ice on all blades & Ice on all blades but one \\
\hline & Frequency/damping & Frequency/damping & Frequency/damping \\
$1^{\text {st }}$ Tower FA & $0.329 \mathrm{~Hz} / 5.59 \%$ & $0.324 \mathrm{~Hz} / 5.13 \%$ & $0.324 \mathrm{~Hz} / 5.24 \%$ \\
$1^{\text {st }}$ Flap Yaw & $0.666 \mathrm{~Hz} / 6.20 \%$ & $0.588 \mathrm{~Hz} / 7.05 \%$ & $0.585 \mathrm{~Hz} / 7.06 \%$ \\
$1^{\text {st }}$ Edge Yaw & $1.059 \mathrm{~Hz} / 0.75 \%$ & $0.948 \mathrm{~Hz} / 0.95 \%$ & $0.948 \mathrm{~Hz} / 0.99 \%$ \\
$2^{\text {nd }}$ Flap Yaw & $1.853 \mathrm{~Hz} / 3.24 \%$ & $1.696 \mathrm{~Hz} / 2.05 \%$ & $1.695 \mathrm{~Hz} / 2.33 \%$ \\
\hline
\end{tabular}

For a qualitative analysis of the signals, the PSD from some sensors is computed and shown in Figure 2. One point along each one of the three blades at the same distance from the rotor center is taken into account.
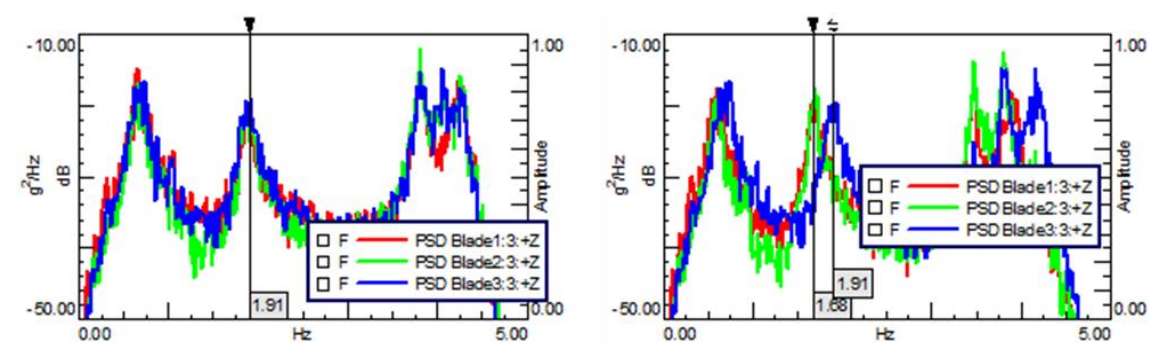

Figure 2: PSD of acceleration measured on one point on each blade in parked conditions. Standard configuration (left) compared to the one with ice on all blades but one (right).

First of all, parked conditions are analyzed. Two different configurations are considered, the standard one and that one with ice formation on all the blades except one. The curves are shown in Figure 2. Almost all peaks for the two blades 
with ice in the plot on the right are shifted to lower frequencies, as a consequence of the mass increase.

Figure 3 and Figure 4 show the PSDs in operating conditions for different configurations. The fundamental harmonic frequency is $0.217 \mathrm{~Hz}$ because of an averaged rotational speed equal to $13.02 \mathrm{rpm}$. The PSD amplitude in correspondence of the first harmonic increases for ice configuration in comparison with the standard configuration; the same considerations can be done regarding the mass unbalance configuration, also if the blade mass is quite bigger than the unbalanced mass and the differences between the two curves are not so evident. The other harmonics can also be identified, but the amplitudes are less high than the first one.
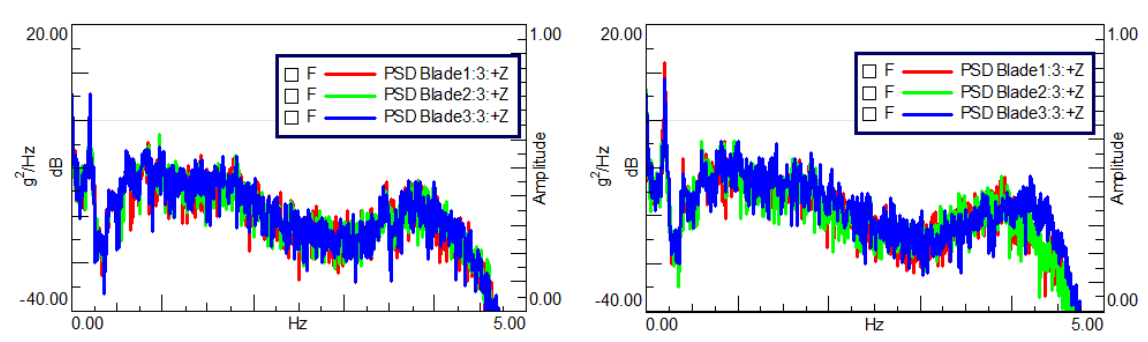

Figure 3: PSDs from acceleration measured on one point on each blade in operating conditions; ice on all the blades (left) compared to ice on all the blades but one (right).
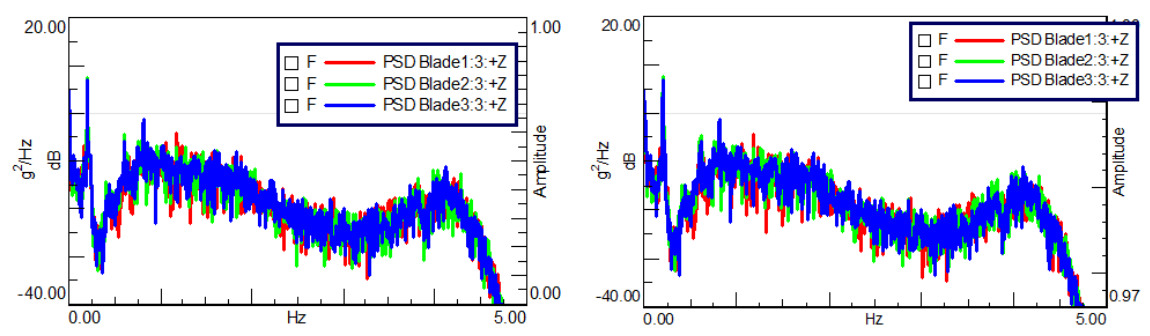

Figure 4: PSDs from acceleration measured on one point on each blade in operating conditions; standard configuration (left) compared to mass unbalance configuration (right)

\section{Conclusions}

In this paper, an advanced OMA technique has been applied to a wind turbine to predict in advance failures or damages with a monitoring application based on the analysis of modal parameters and their variations in operating conditions. The simulation model of a wind turbine has been built using an aero-elastic code; first of all, data have been generated for parked conditions and processed using the 
OMA technique to identify a reference set of modes. Then, different load cases have been considered and data have been processed to analyze the difference in frequency and amplitude from one case to another. For example, adding ice on all the blades causes a frequency shift toward lower frequencies for all the natural frequencies; if on one blade there is no ice formation, the difference between this blade and the other two in terms of PSD can be identified. Then an operating case has been simulated and the data have been processed and analyzed for different conditions, with the icing and with the presence of an unbalanced mass on one of the three blades. In operating conditions, the presence of harmonic components in the signals makes the modal identification process critical because these components have a much higher energy than the ones related to the structural response. In the literature different techniques to separate the components and enhance the identification process have been implemented. In the preliminary study, the harmonics components have not been removed, but in a more detailed study the prediction in advance of failures and damages will be analyzed after using one of the harmonic removal methods.

\section{Acknowledgments}

The authors acknowledge the European Commission (EC) for their research grant under the project FP7-PEOPLE-2012-ITN 309395 "MARE-WINT" (new MAterials and Reliability in offshore WINd Turbines technology), see http://marewint.eu/.

\section{References}

[1] G.H. James III, T.G. Carne, J.P. Lauffer, The Natural Excitation Techniques (NExT) for modal parameters extraction from operating wind turbines, Sandia National Laboratories Technical Report, USA, 1993.

[2] B. Peeters, P. Guillaume, H. Van der Auweraer, B. Cauberghe, P. Verboven, J. Leuridan, Automotive and Aerospace applications of LMS PolyMAX modal parameter estimation method, In Proceedings of IMAC 22, Dearborne, USA, 2004.

[3] B. Peeters, H. Van der Auweraer, PolyMAX: a revolution in Operational Modal Analysis, Proceedings of $1^{\text {st }}$ IOMAC, Copenhagen, Denmark, 2005.

[4] W. Heylen, S. Lemmens, P. Sas, Modal Analysis Theory and Testing, Katholieke Universiteit Leuven, Department Werktuigkunde, Leuven, 1997.

[5] B. Peeters, H. Van der Auweraer, F. Vanhollebeke, P. Guillaume, Operational Modal Analysis for estimating the dynamic properties of a football stadium structure during a football game, Shock and Vibration, 14(4), 2007, 283-303.

[6] J. Debille, S. Pauwels, B. Peeters, The benefits of Operational Modal Analysis of aircraft and spacecraft structures, Proceedings of ETTC, Toulouse, France, 2005.

[7] J. Jonkman, S. Buttefield, W. Musial, G. Scott, Definition of a 5 MW reference wind turbine for offshore system development, NREL/TP-500-38060-Technical Report, USA, 2009.

[8] LMS Samtech Iberica, Samtech for Wind Turbines V3.2.01 Online help, Spain, 2012.

[9] S. Manzato, D. Moccia, B. Peeters, K. Janssens, J.R. White, A review of harmonic removal methods for improved Operational Modal Analysis of wind turbines, Proceedings of ISMA 2012, Leuven, Belgium, 2012. 
[10] M. Luczak, M. Firla, D. Sporna, S. Manzato, B. Peeters, Structural monitoring of the small wind turbine test stand, Proceedings of EURODYN 2011, Leuven, Belgium, 2011.

[11] Germanischer Lloyd WindEnergie GmbH: Guideline for the Certification of Wind Turbines, Edition 2010

[12] LMS International, LMS Test.Lab Rev. 12A User Manual, Belgium, 2012.

[13] C. Hochart, G. Fortin, J. Perron, A. Ilinca, Wind turbine performance under icing conditions, Wind Energ., 11: 319-333, 2008 